\title{
Assessment of a single-operator real-time ultrasound-guided epidural technique in a porcine phantom
}

\author{
Silke Brinkmann, MBBS • Christopher H. Mitchell, FANZCA • \\ Graham Hocking, FANZCA
}

Received: 13 October 2011/Accepted: 16 November 2011/Published online: 2 December 2011

(C) Canadian Anesthesiologists' Society 2011

\section{To the Editor,}

Recent correspondence in the Journal highlighted the usefulness of a porcine carcass phantom for teaching and learning ultrasound-guided paravertebral techniques because of the notable similarity of spinal and paravertebral structures in pigs and humans. ${ }^{1}$ We have previously described a method of deodorizing and curing a porcine model for lumbar sonoanatomy. ${ }^{2}$ We have now used the porcine model to assess the feasibility of a singleoperator ultrasound-guided epidural technique.

The phantom was a T12-L5 section of pork spine with associated musculature cured in $66 \%$ alcoholic gel (Aqium Gel, Ego Pharmaceuticals Pty Ltd, Australia). The Sir Charles Gairdner Group Human Research Ethics Committee approved the project (No. 2010-179), and all participants gave written informed consent. Ten anesthesiologists were recruited who were experienced in ultrasound-guided regional anesthesia but were non-expert in spinal sonography. They were shown a technique of paramedian epidural insertion using ultrasound assistance (Sonosite M-Turbo, 13-8 MHz linear array, Bothel, WA, USA) with a $17 \mathrm{G}$ echogenic Tuohy needle (Pajunk ${ }^{\circledR}$ TuohySono, Geisingen, Germany) and an Epidrum ${ }^{\circledR}$ device (Exmoor Innovations, UK) that provides visual confirmation of loss of resistance (LOR) when the air-filled diaphragm deflates. The Tuohy-Epidrum-syringe assembly was introduced in-plane from the caudal end of the

Parts of the data have been presented as a poster at the Australian New Zealand College of Anaesthetists Annual Scientific Meeting in Hong Kong, May 2011.

S. Brinkmann, MBBS - C. H. Mitchell, FANZCA ·

G. Hocking, FANZCA $(\bowtie)$

Sir Charles Gairdner Hospital, Perth, WA, Australia

e-mail: graham.hocking@anaesthesia.uwa.edu.au transducer, and the needle was advanced until the tip approached the ligamentum flavum. At this point, the Epidrum device was inflated (Figure). Deflation occurred as the needle tip entered the epidural space, and the Epidrum and syringe were removed. The primary endpoint was successful threading of the catheter and appearance in the epidural space at the cephalad end of the phantom. Each anesthesiologist performed the task twice. Their attempts were recorded and timed by one of the authors (S.B.). Participants made a subjective assessment of the technique using a structured questionnaire. Secondary endpoints included time taken from needle first on view to visual LOR and percentage of time the needle was in view. The catheter was threaded successfully in 19 out of 20 attempts. One failure occurred due to premature Epidrum deflation prior to the needle entering the epidural space. This was clearly imaged but protocol dictated an attempt at catheter insertion. Mean (standard deviation) time to LOR was 51 (27) sec. The needle was visible $90(10) \%$ of the time corresponding with $90 \%$ of participants assessing visibility as excellent on a three-point rating scale. The task was evaluated as easy or intermediate by $60 \%$ of participants. While the other $40 \%$ of participants performed equally well, they expressed their natural caution over a new technique that others have labelled as "advanced". All participants would consider performing the technique on a patient, which suggests that phantom practice allowed participants to gain confidence that could result in changes to clinical practice. The echogenic TuohySono overcame the needle visibility difficulties at steep insertion angles that have previously been highlighted. ${ }^{3}$ Stiffness of the Tuohy allowed the needle rather than the transducer to be manipulated to preserve an in-plane view. The stationary transducer maintained an optimal image of the target at all times. The Epidrum made a single-operator 
Figure The epidural needle placed near the ligamentum flavum with the Epidrum device inflated
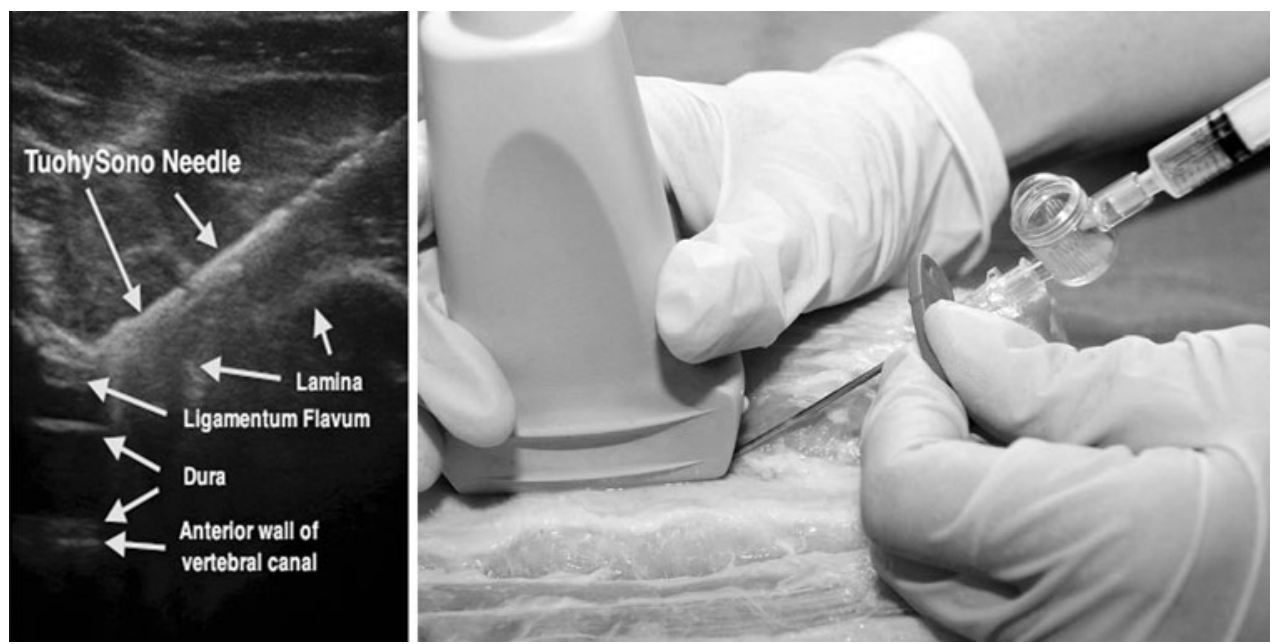

ultrasound-assisted technique possible. However, the operator had to focus on the Epidrum for visual confirmation of LOR as the TuohySono advanced through the ligamentum flavum. Therefore, at this stage, the ultrasound probe could have been put down and conventional LOR techniques used. We acknowledge the usefulness and popularity of curvilinear transducers in spinal sonoanatomy, but the wide linear array provided optimal definition of the target interspace and improved needle visualization because of higher frequency and narrower beam thickness.

Ultrasound-guided neuraxial blocks have been considered advanced techniques due to poor needle visibility or the requirement for complicated needle guidance devices. $^{3,4}$ Our porcine model provided a high-fidelity inexpensive learning tool in the same way as previously described for practising paravertebral and epidural techniques. $^{1,2,5}$ The reality of the model is limited by prone positioning, air tracking, echotexture, and slight anatomical differences. However, the porcine phantom allowed us to demonstrate that our technique is both feasible and highly acceptable to anesthesiologists. The participants gained competency on the model and were willing to apply their new skills to clinical practice. We have subsequently used the technique successfully in a small number of obstetric patients, and further clinical studies are underway.
Disclosures Dr. Mitchell designed the Pajunk TuohySono needle but was excluded from participating in data collection. The other authors have nothing to disclose.

Finance Departmental funding

Competing interests None declared.

\section{References}

1. Abdallah $F W$, Hocking $G$, Chan $V W$. Porcine carcass phantom for ultrasound imaging and hands-on practice in needle insertion in the thoracic paravertebral space. Can J Anesth 2011; 58: 875-7.

2. Hocking G, Gilmour F, Michell C. Porcine phantom for ultrasound-guided neuraxial blockade. Br J Anaesth 2010 (E-letter 28 January 2010). Available from URL: http://bja.oxfordjournals. org/forum/topic/brjana_el\%3B5535 (accessed November 2011).

3. Karmakar MK, Li X, Ho AM, Kwok WH, Chui PT. Real-time ultrasound-guided paramedian epidural access: evaluation of a novel in-plane technique. Br J Anaesth 2009; 102: 845-54.

4. Tran D, Kamani AA, Al-Attas E, Lessoway VA, Massey S, Rohling $R N$. Single-operator real-time ultrasound-guidance to aim and insert a lumbar epidural needle. Can J Anesth 2010; 57: 313-21.

5. Hocking G, Hebard S, Mitchell CH. A review of the benefits and pitfalls of phantoms in ultrasound-guided regional anesthesia. Reg Anesth Pain Med 2011; 36: 162-70. 